\title{
UNIFORM APPROXIMATION ON IDEALS OF MULTILINEAR MAPPINGS
}

\author{
GERALDO BOTELHO, PABLO GALINDO and LEONARDO PELLEGRINI*
}

\begin{abstract}
For each ideal of multilinear mappings $\mathscr{M}$ we explicitly construct a corresponding ideal ${ }^{a} \mathcal{M}$ such that multilinear forms in ${ }^{a} \mathcal{M}$ are exactly those which can be approximated, in the uniform norm, by multilinear forms in $\mathscr{M}$. This construction is then applied to finite type, compact, weakly compact and absolutely summing multilinear mappings. It is also proved that the correspondence $\mathscr{M} \mapsto{ }^{a} \mathscr{M}$ is Aron-Berner stability preserving.
\end{abstract}

\section{Introduction}

The theory of ideals of multilinear mappings (multi-ideals) between Banach spaces (see [6], [7], [8], [9], [10], [11], [16], [17], [18], [19], [24], [25] and references therein) studies, as in the theory of linear operator ideals, plenty of non-closed ideals. So the question of describing their closures in the uniform norm is quite natural. Our main aim is to construct an approximation scheme for multi-ideals similar to the H. Jarchow and A. Pełczyński description of the closed injective hull of an operator ideal, which can be found in [21, Section 20.7].

Given a multi-ideal $\mathscr{M}$, we construct a corresponding multi-ideal ${ }^{a} \mathcal{M}$ such that every multilinear form in ${ }^{a} \mathcal{M}$ can be approximated, in the uniform norm, by multilinear forms in $\mathcal{M}$. For multilinear mappings taking values in a Banach space $F$ the approximation takes place, like in the linear case, in the larger space $\ell_{\infty}\left(B_{F^{*}}\right)$. We do not only prove the existence of such multi-ideal ${ }^{a} \mathcal{M}$, we give it an explicit description that roughly speaking means that a multilinear mapping $A$ can be approximated by elements in $\mathcal{M}$ if the norm of any sum of images of $A$ is almost "dominated" by the norm of the sum of the corresponding images by some element in $\mathscr{M}$, (see Definition 2.1). Relying on such description we obtain several properties of ${ }^{a} \mathscr{M}$. This is done in Section 2.

It should be noted that, as usual, there are several a priori possibilities to transpose the Jarchow-Pełczyński construction to the multilinear case. The dir-

\footnotetext{
* The first author was supported by CNPq Project 202162/2006-0, the second author partially by MEC-FEDER Project MTM2007-64521, and the third author by ProIP Project-USP.

Received 19 September 2008.
} 
ect and most obvious transposition simply does not work. So an important step was to identify, among all possible multilinear generalizations of the JarchowPełczyński construction, the one that performs the desired approximation. The results we prove show that we have selected the correct definition.

The rest of paper is organized as follows. Section 1 provides the basic notions and fixes the notation. In Section 3 we apply the approximation theorem to finite type and compact mappings. Denoting the multi-ideal of multilinear mappings of finite type by $\mathscr{L}_{f}$, we prove that its corresponding ideal ${ }^{a}\left(\mathscr{L}_{f}\right)$ coincides, modulo the approximation property, with the extensively studied (see [3] or [15] for instance) class of multilinear mappings which are weakly continuous on bounded sets. The case of weakly compact and absolutely summing mappings is studied in Section 4, where we prove multilinear counterparts of some important linear results. In Section 5 we show that the correspondence $\mathscr{M} \mapsto{ }^{a} \mathcal{M}$ preserves the stability of $\mathcal{M}$ with respect to Aron-Berner extensions of multilinear mappings to the bidual spaces; this will extend to the multilinear setting known results about bitranspose linear operators. A contribution to the linear theory is also obtained.

\section{Background and notation}

Throughout $n$ is a positive integer, $E, E_{1}, \ldots, E_{n}, F, G_{1}, \ldots, G_{n}$ and $H$ are (real or complex) Banach spaces. $\mathscr{L}(E ; F)$ denotes the Banach space, endowed with the usual sup norm, of bounded linear operators from $E$ to $F$ and $\mathscr{L}\left(E_{1}, \ldots, E_{n} ; F\right)$ the Banach space, endowed with the usual sup norm, of continuous $n$-linear mappings from $E_{1} \times \cdots \times E_{n}$ to $F$. If $F$ is the scalar field we simply write $E^{*}$ and $\mathscr{L}\left(E_{1}, \ldots, E_{n}\right)$. If $E_{1}=\cdots=E_{n}=E$ we write $\mathscr{L}\left({ }^{n} E ; F\right)$ and $\mathscr{L}\left({ }^{n} E\right)$. Linear combinations of mappings of the form $A\left(x_{1}, \ldots, x_{n}\right)=\varphi_{1}\left(x_{1}\right) \cdots \varphi_{n}\left(x_{n}\right) b$, where $\varphi_{j} \in E_{j}^{*}$ and $b \in F$, are called $n$-linear mappings of finite type. The space of all such mappings is denoted by $\mathscr{L}_{f}\left(E_{1}, \ldots, E_{n} ; F\right)$. The mappings belonging to its closure $\overline{\mathscr{L}_{f}}$ are called approximable. For each $A \in \mathscr{L}\left(E_{1}, \ldots, E_{n} ; F\right)$, we denote by $A_{L} \in \mathscr{L}\left(E_{1} \widehat{\otimes}_{\pi} \cdots \widehat{\otimes}_{\pi} E_{n} ; F\right)$ its linearization on the completed $n$-fold projective tensor product that is defined by

$$
A_{L}\left(x_{1} \otimes \cdots \otimes x_{n}\right)=A\left(x_{1}, \ldots, x_{n}\right) \quad \text { for all } \quad x_{j} \in E_{j} .
$$

For the general theory of multilinear mappings between Banach spaces we refer to S. Dineen [15]

DEFINITION 1.1 (Ideals of multilinear mappings or multi-ideals). An ideal of $n$-linear mappings (or $n$-ideal) $\mathscr{M}$ is a subclass of the class of all continuous $n$-linear mappings between Banach spaces such that for Banach spaces 
$E_{1}, \ldots, E_{n}$ and $F$, the components $\mathscr{M}\left(E_{1}, \ldots, E_{n} ; F\right):=\mathscr{L}\left(E_{1}, \ldots\right.$, $\left.E_{n} ; F\right) \cap \mathscr{M}$ satisfy:

(i) $\mathscr{M}\left(E_{1}, \ldots, E_{n} ; F\right)$ is a linear subspace of $\mathscr{L}\left(E_{1}, \ldots, E_{n} ; F\right)$ which contains the $n$-linear mappings of finite type.

(ii) The ideal property: if $A \in \mathscr{M}\left(E_{1}, \ldots, E_{n} ; F\right), u_{j} \in \mathscr{L}\left(G_{j} ; E_{j}\right)$ for $j=$ $1, \ldots, n$ and $t \in \mathscr{L}(F ; H)$, then $t \circ A \circ\left(u_{1}, \ldots, u_{n}\right)$ is in $\mathcal{M}\left(G_{1}, \ldots\right.$, $\left.G_{n} ; H\right)$.

If there is a function $\|\cdot\| \mu: M \longrightarrow \mathrm{R}^{+}$satisfying

(i') There is $0<p \leq 1$ such that $\|\cdot\|_{M}$ restricted to $\mathscr{M}\left(E_{1}, \ldots, E_{n} ; F\right)$ is a $p$-norm for all Banach spaces $E_{1}, \ldots, E_{n}$ and $F$,

(ii') $\left\|A: \mathrm{K}^{n} \longrightarrow \mathrm{K}: A\left(\lambda_{1}, \ldots, \lambda_{n}\right)=\lambda_{1} \ldots \lambda_{n}\right\|_{\mathscr{M}}=1$ for all $n$,

(iii') If $A \in \mathscr{M}\left(E_{1}, \ldots, E_{n} ; F\right), u_{j} \in \mathscr{L}\left(G_{j} ; E_{j}\right)$ for $j=1, \ldots, n$ and $t \in \mathscr{L}(F ; H)$, then $\left\|t \circ A \circ\left(u_{1}, \ldots, u_{n}\right)\right\|_{\mathcal{M}} \leq\|t\|\|A\|_{\mathcal{M}}\left\|u_{1}\right\| \ldots\left\|u_{n}\right\|$, then $\mathscr{M}$ is called a quasi-normed (normed if $p=1$ ) n-ideal. Quasi-Banach (Banach if $p=1) n$-ideals are defined in the obvious way. A (Banach) multiideal is a sequence $\left(\mathscr{M}_{n}\right)_{n=1}^{\infty}$ where each $\mathscr{M}_{n}$ is a (Banach) $n$-ideal.

When $n=1$ we recover the classical theory of operator ideals, for which the reader is referred to [13].

A Banach $n$-ideal $\mathscr{M}$ is said to be closed if each component $\mathscr{M}\left(E_{1}, \ldots\right.$, $\left.E_{n} ; F\right)$ is a (sup-norm) closed subspace of $\mathscr{L}\left(E_{1}, \ldots, E_{n} ; F\right)$. A Banach multi-ideal $\left(\mathscr{M}_{n}\right)_{n=1}^{\infty}$ is closed if each $\mathscr{M}_{n}$ is closed.

An $n$-ideal $\mathscr{M}$ is injective if whenever $A \in \mathscr{L}\left(E_{1}, \ldots, E_{n} ; F\right), i: F \longrightarrow$ $G$ is an isometric embedding and $i \circ A \in \mathcal{M}\left(E_{1}, \ldots, E_{n} ; G\right)$, then $A \in$ $\mathscr{M}\left(E_{1}, \ldots, E_{n} ; F\right)$.

\section{The approximation scheme}

Definition 2.1. Let $\mathscr{M}$ be an $n$-ideal. A mapping $A \in \mathscr{L}\left(E_{1}, \ldots, E_{n} ; F\right)$ is said to be $\mathscr{M}$-approximable, in symbols $A \in{ }^{a} \mathcal{M}\left(E_{1}, \ldots, E_{n} ; F\right)$, if there are a Banach space $G$ and an $n$-linear mapping $B \in \mathscr{M}\left(E_{1}, \ldots, E_{n} ; G\right)$ such that for every $\varepsilon>0$ there is $K_{\varepsilon}>0$ such that

$$
\left\|\sum_{i=1}^{k} A\left(x_{i}^{1}, \ldots, x_{i}^{n}\right)\right\| \leq K_{\varepsilon}\left\|\sum_{i=1}^{k} B\left(x_{i}^{1}, \ldots, x_{i}^{n}\right)\right\|+\varepsilon \sum_{i=1}^{k}\left\|x_{i}^{1}\right\| \ldots\left\|x_{i}^{n}\right\|,
$$

for every $k \in \mathrm{N}$ and any $x_{i}^{j} \in E_{j}, j=1, \ldots, n, i=1, \ldots, k$.

Remark 2.2. (a) It is obvious from the definition that, for every $n$-ideal $\mathscr{M}$, ${ }^{a} \mathcal{M}$ is injective. It is also obvious that ${ }^{a} \mathscr{M} \subseteq{ }^{a} \mathcal{N}$ if $\mathscr{M} \subseteq \mathscr{N}$. 
(b) Let us see that ${ }^{a}\left({ }^{a} \mathcal{M}\right)={ }^{a} \mathcal{M}$ : it is clear that ${ }^{a} \mathcal{M}$ contains $\mathscr{M}$, so ${ }^{a} \mathcal{M} \subseteq{ }^{a}\left({ }^{a} \mathcal{M}\right)$. For the converse, given $A \in{ }^{a}\left({ }^{a} \mathscr{M}\right)\left(E_{1}, \ldots, E_{n} ; F\right)$, there are a Banach space $G$ and an $n$-linear mapping $B \in \mathscr{M}^{a}\left(E_{1}, \ldots, E_{n} ; G\right)$ such that for every $\varepsilon>0$ there is $K_{\varepsilon}$ such that

$$
\left\|\sum_{i=1}^{k} A\left(x_{i}^{1}, \ldots, x_{i}^{n}\right)\right\| \leq K_{\varepsilon}\left\|\sum_{i=1}^{k} B\left(x_{i}^{1}, \ldots, x_{i}^{n}\right)\right\|+\varepsilon \sum_{i=1}^{k}\left\|x_{i}^{1}\right\| \ldots\left\|x_{i}^{n}\right\|,
$$

for every $k \in \mathrm{N}$ and any $x_{i}^{j} \in E_{j}, j=1, \ldots, n, i=1, \ldots, k$. Since $B \in{ }^{a} \mathcal{M}$, there are a Banach space $G$ and an $n$-linear mapping $B \in a \mathscr{M}\left(E_{1}, \ldots, E_{n} ; G\right)$ such that for every $\varepsilon>0$ there is $N_{\varepsilon}$ such that

$$
\left\|\sum_{i=1}^{k} B\left(x_{i}^{1}, \ldots, x_{i}^{n}\right)\right\| \leq N_{\varepsilon}\left\|\sum_{i=1}^{k} C\left(x_{i}^{1}, \ldots, x_{i}^{n}\right)\right\|+\varepsilon \sum_{i=1}^{k}\left\|x_{i}^{1}\right\| \ldots\left\|x_{i}^{n}\right\|,
$$

for every $k \in \mathrm{N}$ and any $x_{i}^{j} \in E_{j}, j=1, \ldots, n, i=1, \ldots, k$. Given $\varepsilon>0$, one easily checks that for every $k \in \mathrm{N}$ and any $x_{i}^{j} \in E_{j}, j=1, \ldots, n$, $i=1, \ldots, k$,

$$
\left\|\sum_{i=1}^{k} A\left(x_{i}^{1}, \ldots, x_{i}^{n}\right)\right\| \leq L_{\varepsilon}\left\|\sum_{i=1}^{k} C\left(x_{i}^{1}, \ldots, x_{i}^{n}\right)\right\|+\varepsilon \sum_{i=1}^{k}\left\|x_{i}^{1}\right\| \ldots\left\|x_{i}^{n}\right\|,
$$

where $L_{\varepsilon}$ may be chosen as $K_{\frac{\varepsilon}{2}} \cdot N_{\frac{\varepsilon}{2 K \frac{\varepsilon}{2}}}$. Thus, $A \in{ }^{a} \mathcal{M}\left(E_{1}, \ldots, E_{n} ; F\right)$.

(c) For operator ideals, this is the Jarchow-Pełczyński description of the closed injective hull of a given operator ideal (see [21, Theorem 20.7.3]). In particular, when $n=1$ and $\mathcal{M}$ is the ideal $\Pi_{p}$ of absolutely $p$-summing operators, $1 \leq p<+\infty$, we get ${ }^{a} \mathcal{M}=\mathscr{H}$, where $\mathscr{H}$ is the ideal of absolutely continuous linear operators (see [14, Chapter 15]).

Proposition 2.3. If $\mathcal{M}$ is a Banach $n$-ideal, then ${ }^{a} \mathcal{M}$ is a closed injective $n$-ideal containing $\mathcal{M}$.

Proof. Since ${ }^{a} \mathcal{M}$ contains $\mathcal{M}$, it also contains the $n$-linear mappings of finite type.

Given $A_{1}, A_{2} \in a \mathcal{M}\left(E_{1}, \ldots, E_{n} ; F\right)$, let $B_{j} \in \mathcal{M}\left(E_{1}, \ldots, E_{n} ; G_{j}\right), j=$ 1,2 , for which the definition holds. Define $G:=G_{1} \oplus_{1} G_{2}$ and $B \in \mathscr{L}\left(E_{1}, \ldots\right.$, $\left.E_{n} ; G\right)$ by $B\left(x_{1}, \ldots, x_{n}\right)=\left(B_{1}\left(x_{1}, \ldots, x_{n}\right), B_{2}\left(x_{1}, \ldots, x_{n}\right)\right)$. $B$ belongs to $\mathcal{M}$ as $B=i_{i} \circ B_{1}+i_{2} \circ B_{2}$, where $i_{j}$ are the canonical inclusions. It is a routine computation to verifiy that such $B$ fulfills the conditions of the definition for $A_{1}+A_{2}$. Thus $A_{1}+A_{2} \in{ }^{a} \mathcal{M}\left(E_{1}, \ldots, E_{n} ; F\right)$.

The ideal property is easily checked. 
Let $\left(A_{j}\right)_{j=1}^{\infty} \subseteq{ }^{a} \mathcal{M}\left(E_{1}, \ldots, E_{n} ; F\right), A_{j} \longrightarrow A \in \mathscr{L}\left(E_{1}, \ldots, E_{n} ; F\right)$ in norm. For each $j \in \mathrm{N}$, take $G_{j}$ and $0 \neq B_{j} \in \mathscr{M}\left(E_{1}, \ldots, E_{n} ; G_{j}\right)$ associated to $A_{j}$ according to the definition. Define $G:=\left(\bigoplus_{j=1}^{\infty} G_{j}\right)_{1}$ and for each $j$ consider the canonical inclusion $i_{j}: G_{j} \longrightarrow G$. It is clear that each $i_{j} \circ B_{j}$ belongs to $\mathcal{M}$. Since

$$
\sum_{j=1}^{\infty} \frac{1}{2^{j}\left\|B_{j}\right\|_{\mathscr{M}}}\left\|i_{j} \circ B_{j}\right\|_{\mathscr{M}} \leq \sum_{j=1}^{\infty} \frac{1}{2^{j}\left\|B_{j}\right\|_{\mathcal{M}}}\left\|i_{j}\right\|\left\|B_{j}\right\|_{\mathscr{M}}=\sum_{j=1}^{\infty} \frac{1}{2^{j}}<+\infty,
$$

and $\left(\mathcal{M}\left(E_{1}, \ldots, E_{n} ; G\right),\|\cdot\|_{\mathscr{M}}\right)$ is a Banach space, the series $\sum_{j=1}^{\infty} \frac{1}{2^{j}\left\|B_{j}\right\|_{\mathscr{M}}} i_{j}$ 。 $B_{j}$ converges in this space. Call

$$
B:=\sum_{j=1}^{\infty} \frac{1}{2^{j}\left\|B_{j}\right\|_{\mathscr{M}}} i_{j} \circ B_{j} \in \mathcal{M}\left(E_{1}, \ldots, E_{n} ; G\right) .
$$

From $\|\cdot\| \leq\|\cdot\|_{\mathscr{M}}$ (see [9, Satz 2.2.5]) it follows that such series is pointwise convergent. Given $\varepsilon>0$, let $j_{0} \in \mathrm{N}$ be such that $\left\|A_{j_{0}}-A\right\|<\frac{\varepsilon}{2}$. Take $K$ such that for every $k \in \mathrm{N}$ and any $x_{i}^{j} \in E_{j}, j=1, \ldots, n, i=1, \ldots, k$,

$$
\left\|\sum_{i=1}^{k} A_{j_{0}}\left(x_{i}^{1}, \ldots, x_{i}^{n}\right)\right\| \leq K\left\|\sum_{i=1}^{k} B_{j_{0}}\left(x_{i}^{1}, \ldots, x_{i}^{n}\right)\right\|+\frac{\varepsilon}{2} \sum_{i=1}^{k}\left\|x_{i}^{1}\right\| \ldots\left\|x_{i}^{n}\right\| .
$$

Then, for every $k \in \mathrm{N}$ and any $x_{i}^{j} \in E_{j}, j=1, \ldots, n, i=1, \ldots, k$,

$$
\begin{aligned}
& \left\|\sum_{i=1}^{k} A\left(x_{i}^{1}, \ldots, x_{i}^{n}\right)\right\| \\
& \leq\left\|\sum_{i=1}^{k}\left(A-A_{j_{0}}\right)\left(x_{i}^{1}, \ldots, x_{i}^{n}\right)\right\|+\left\|\sum_{i=1}^{k} A_{j_{0}}\left(x_{i}^{1}, \ldots, x_{i}^{n}\right)\right\| \\
& \quad \leq K\left\|\sum_{i=1}^{k} B_{j_{0}}\left(x_{i}^{1}, \ldots, x_{i}^{n}\right)\right\|+\varepsilon \sum_{i=1}^{k}\left\|x_{i}^{1}\right\| \ldots\left\|x_{i}^{n}\right\| \\
& \quad=K 2^{j_{0}}\left\|B_{j_{0}}\right\|_{\mathscr{M}}\left\|\sum_{i=1}^{k} \frac{B_{j_{0}}\left(x_{i}^{1}, \ldots, x_{i}^{n}\right)}{2^{j_{0}}\left\|B_{j_{0}}\right\| \mathscr{M}}\right\|+\varepsilon \sum_{i=1}^{k}\left\|x_{i}^{1}\right\| \ldots\left\|x_{i}^{n}\right\| \\
& \quad \leq K 2^{j_{0}}\left\|B_{j_{0}}\right\| \sum_{j=1}^{\infty}\left\|\sum_{i=1}^{k} \frac{B_{j}\left(x_{i}^{1}, \ldots, x_{i}^{n}\right)}{2^{j}\left\|B_{j}\right\|_{\mathscr{M}}}\right\|+\varepsilon \sum_{i=1}^{k}\left\|x_{i}^{1}\right\| \ldots\left\|x_{i}^{n}\right\| \\
& \quad \leq K 2^{j_{0}}\left\|B_{j_{0}}\right\| \mathscr{M}\left\|\sum_{i=1}^{k} B\left(x_{i}^{1}, \ldots, x_{i}^{n}\right)\right\|+\varepsilon \sum_{i=1}^{k}\left\|x_{i}^{1}\right\| \ldots\left\|x_{i}^{n}\right\| .
\end{aligned}
$$


It results that $A$ belongs to ${ }^{a} \mathcal{M}$, completing the proof.

For every Banach space $E$, by $i_{E}$ we mean the canonical isometric embedding $E \longrightarrow \ell_{\infty}\left(B_{E^{*}}\right)$. Besides of performing the desired approximation scheme, next result shows that ${ }^{a} \mathscr{M}$ could have been defined by a condition which seems to be less demanding at first glance.

THEOREm 2.4. Let $\mathscr{M}$ be an n-ideal. The following are equivalent for an $n$-linear mapping $A \in \mathscr{L}\left(E_{1}, \ldots, E_{n} ; F\right)$ :

(a) $A \in{ }^{a} M\left(E_{1}, \ldots, E_{n} ; F\right)$.

(b) For every $\varepsilon>0$ there is an n-linear mapping $C \in \mathscr{M}\left(E_{1}, \ldots, E_{n}\right.$; $\left.\ell_{\infty}\left(B_{F^{*}}\right)\right)$ such that $\left\|i_{F} \circ A-C\right\|<\varepsilon$.

(c) There is a set $\Gamma$ and an isometric embedding $i_{F}^{\Gamma}: F \longrightarrow \ell_{\infty}(\Gamma)$ such that for every $\varepsilon>0$ there is an n-linear mapping $C \in \mathcal{M}\left(E_{1}, \ldots, E_{n}\right.$; $\left.\ell_{\infty}(\Gamma)\right)$ such that $\left\|i_{F}^{\Gamma} \circ A-C\right\|<\varepsilon$.

(d) For every $\varepsilon>0$ there are a Banach space $G_{\varepsilon}$ and an $n$-linear mapping $B_{\varepsilon} \in \mathscr{M}\left(E_{1}, \ldots, E_{n} ; G_{\varepsilon}\right)$ such that

$$
\left\|\sum_{i=1}^{k} A\left(x_{i}^{1}, \ldots, x_{i}^{n}\right)\right\| \leq\left\|\sum_{i=1}^{k} B_{\varepsilon}\left(x_{i}^{1}, \ldots, x_{i}^{n}\right)\right\|+\varepsilon \sum_{i=1}^{k}\left\|x_{i}^{1}\right\| \ldots\left\|x_{i}^{n}\right\|,
$$

for every $k \in \mathrm{N}$ and any $x_{i}^{j} \in E_{j}, j=1, \ldots, n, i=1, \ldots, k$.

Proof. (d) $\Longrightarrow$ (b) Let $\varepsilon>0$ be given. By assumption, there are $G_{\varepsilon}$ and $B_{\varepsilon} \in \mathcal{M}\left(E_{1}, \ldots, E_{n} ; G_{\varepsilon}\right)$ such that for every $k \in \mathrm{N}$ and any $x_{i}^{j} \in E_{j}, j=$ $1, \ldots, n, i=1, \ldots, k$,

$$
\left\|\sum_{i=1}^{k} A\left(x_{i}^{1}, \ldots, x_{i}^{n}\right)\right\| \leq\left\|\sum_{i=1}^{k} B_{\varepsilon}\left(x_{i}^{1}, \ldots, x_{i}^{n}\right)\right\|+\varepsilon \sum_{i=1}^{k}\left\|x_{i}^{1}\right\| \ldots\left\|x_{i}^{n}\right\| .
$$

So we have for their respective linearizations $A_{L}$ and $\left(B_{\varepsilon}\right)_{L}$,

$$
\left\|A_{L}\left(\sum_{i=1}^{k} x_{i}^{1} \otimes \cdots \otimes x_{i}^{n}\right)\right\| \leq\left\|\left(B_{\varepsilon}\right)_{L}\left(\sum_{i=1}^{k} x_{i}^{1} \otimes \cdots \otimes x_{i}^{n}\right)\right\|+\varepsilon \sum_{i=1}^{k}\left\|x_{i}^{1}\right\| \ldots\left\|x_{i}^{n}\right\|,
$$

for every $\sum_{i=1}^{k} x_{i}^{1} \otimes \cdots \otimes x_{i}^{n} \in E_{1} \otimes_{\pi} \cdots \otimes_{\pi} E_{n}$. Taking the infimum over all representations of a tensor $\theta \in E_{1} \otimes_{\pi} \cdots \otimes_{\pi} E_{n}$ in the form $\theta=\sum_{i=1}^{k} x_{i}^{1} \otimes$ $\cdots \otimes x_{i}^{n}$ it results that $\left\|A_{L}(\theta)\right\| \leq\left\|\left(B_{\varepsilon}\right)_{L}(\theta)\right\|+\varepsilon \pi(\theta)$ for every $\theta \in E_{1} \otimes_{\pi}$ $\cdots \otimes_{\pi} E_{n}$. Since $A_{L}$ and $\left(B_{\varepsilon}\right)_{L}$ are continuous, this inequality also holds on $E_{1} \widehat{\otimes}_{\pi} \cdots \widehat{\otimes}_{\pi} E_{n}$. Consider the following linear operator:

$a_{\varepsilon}: E_{1} \otimes_{\pi} \cdots \otimes_{\pi} E_{n} \longrightarrow\left(G_{\varepsilon} \oplus\left(E_{1} \otimes_{\pi} \cdots \otimes_{\pi} E_{n}\right)\right)_{1}, a_{\varepsilon}(\theta)=\left(\left(B_{\varepsilon}\right)_{L}(\theta), \varepsilon \theta\right)$. 
The fact that $a_{\varepsilon}$ is injective allows us to define a linear operator $b_{\varepsilon}: \operatorname{Range}\left(a_{\varepsilon}\right)$ $\longrightarrow F$ by $b_{\varepsilon}\left(a_{\varepsilon}(\theta)\right)=A_{L}(\theta)$. From

$$
\| b_{\varepsilon}\left(a_{\varepsilon}(\theta)\|=\| A_{L}(\theta)\|\leq\|\left(B_{\varepsilon}\right)_{L}(\theta)\|+\varepsilon \pi(\theta)=\| a_{\varepsilon}(\theta) \|,\right.
$$

we find that $\left\|b_{\varepsilon}\right\| \leq 1$. As a continuous linear operator from the normed space Range $\left(a_{\varepsilon}\right)$ into the injective Banach space $\ell_{\infty}\left(B_{F^{*}}\right), i_{F} \circ b_{\varepsilon}$ can be extended to a continuous linear operator $\overline{i_{F} \circ b_{\varepsilon}}:\left(G_{\varepsilon} \oplus\left(E_{1} \otimes_{\pi} \cdots \otimes_{\pi} E_{n}\right)\right)_{1} \longrightarrow \ell_{\infty}\left(B_{F^{*}}\right)$, $\left\|\overline{i_{F} \circ b_{\varepsilon}}\right\| \leq 1$. Define $C \in \mathscr{L}\left(E_{1}, \ldots, E_{n} ; \ell_{\infty}\left(B_{F^{*}}\right)\right)$ by

$$
C\left(x_{1}, \ldots, x_{n}\right)=\overline{i_{F} \circ b_{\varepsilon}}\left(B_{\varepsilon}\left(x_{1}, \ldots, x_{n}\right), 0\right) .
$$

If $j_{\varepsilon}: G_{\varepsilon} \longrightarrow\left(G_{\varepsilon} \oplus\left(E_{1} \otimes_{\pi} \cdots \otimes_{\pi} E_{n}\right)\right)_{1}$ is given by $j_{\varepsilon}(y)=(y, 0)$, then $C=\overline{i_{F} \circ b_{\varepsilon}} \circ j_{\varepsilon} \circ B_{\varepsilon}$, hence $C \in \mathscr{M}\left(E_{1}, \ldots, E_{n} ; \ell_{\infty}\left(B_{F^{*}}\right)\right)$. For $\left(x_{1}, \ldots, x_{n}\right) \in$ $E_{1} \times \cdots \times E_{n}$

$$
\begin{aligned}
i_{F} \circ A_{L}\left(x_{1} \otimes \cdots \otimes x_{n}\right) & =i_{F}\left(b_{\varepsilon}\left(a_{\varepsilon}\left(x_{1} \otimes \cdots \otimes x_{n}\right)\right)\right) \\
& =\overline{i_{F} \circ b_{\varepsilon}}\left(\left(\left(B_{\varepsilon}\right)_{L}\left(x_{1} \otimes \cdots \otimes x_{n}\right), \varepsilon x_{1} \otimes \cdots \otimes x_{n}\right)\right) \\
& =C\left(x_{1}, \ldots, x_{n}\right)+\overline{i_{F} \circ b_{\varepsilon}}\left(\left(0, \varepsilon x_{1} \otimes \cdots \otimes x_{n}\right)\right) .
\end{aligned}
$$

Thus,

$$
\begin{aligned}
\left\|i_{F} \circ A\left(x_{1}, \ldots, x_{n}\right)-C\left(x_{1}, \ldots, x_{n}\right)\right\| & =\left\|\overline{i_{F} \circ b_{\varepsilon}}\left(\left(0, \varepsilon x_{1} \otimes \cdots \otimes x_{n}\right)\right)\right\| \\
& \leq\left\|\overline{i_{F} \circ b_{\varepsilon}}\right\| \varepsilon \pi\left(x_{1} \otimes \cdots \otimes x_{n}\right) \\
& \leq \varepsilon\left\|x_{1}\right\| \ldots\left\|x_{n}\right\|,
\end{aligned}
$$

which proves that $\left\|i_{F} \circ A-C\right\| \leq \varepsilon$.

(c) $\Longrightarrow$ (a) The assumption assures that $i_{F}^{\Gamma} \circ A$ belongs to the sup-norm closure of $\mathscr{M}\left(E_{1}, \ldots, E_{n} ; \ell_{\infty}(\Gamma)\right)$. It follows easily from the definition of ${ }^{a} \mathscr{M}$ that $\bar{M} \subseteq{ }^{a} \mathcal{M}$, therefore $i_{F}^{\Gamma} \circ A \in{ }^{a} \mathcal{M}\left(E_{1}, \ldots, E_{n} ; \ell_{\infty}(\Gamma)\right)$. The injectivity of ${ }^{a} \mathscr{M}$ gives $\left.A \in{ }^{a} \mathscr{M}\left(E_{1}, \ldots, E_{n} ; F\right)\right)$.

The proof is complete as (a) $\Longrightarrow$ (d) and (b) $\Longrightarrow$ (c) are obvious.

Remark 2.5. Given an $n$-ideal $\mathscr{M}$, it follows immediately from Theorem 2.4 that, for every $E_{1}, \ldots, E_{n}$ and $F$,

$$
\begin{aligned}
{ }^{a} \mathcal{M}\left(E_{1}, \ldots, E_{n} ; F\right) & \\
= & \left\{A \in \mathscr{L}\left(E_{1}, \ldots, E_{n} ; F\right): i_{F} \circ A \in \overline{\mathcal{M}\left(E_{1}, \ldots, E_{n} ; \ell_{\infty}\left(B_{F^{*}}\right)\right)}\right\} \\
= & \left\{A \in \mathscr{L}\left(E_{1}, \ldots, E_{n} ; F\right): i_{F}^{\Gamma} \circ A \in \overline{\mathcal{M}\left(E_{1}, \ldots, E_{n} ; \ell_{\infty}(\Gamma)\right)}\right. \\
& \left.\quad \text { or some } \Gamma \text { and some isometric embedding } i_{F}^{\Gamma}: F \longrightarrow \ell_{\infty}(\Gamma)\right\} .
\end{aligned}
$$

In the proof of Theorem 2.4, if $F$ is injective there is no need to go to the larger space $\ell_{\infty}\left(B_{F^{*}}\right)$. 
Corollary 2.6. Let $M$ be an n-ideal. If $F$ is an injective Banach space, then ${ }^{a} \mathcal{M}\left(E_{1}, \ldots, E_{n} ; F\right)=\bar{M}\left(E_{1}, \ldots, E_{n} ; F\right)$ for every $E_{1}, \ldots, E_{n}$. In par-

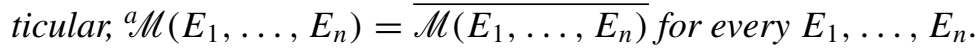

Theorem 2.7. Let $\mathcal{M}$ be a Banach $n$-ideal. $\mathcal{M}={ }^{a} \mathcal{M}$ if and only if $\mathscr{M}$ is closed and injective. Furthermore, ${ }^{a} \mathcal{M}$ is the smallest closed injective $n$-ideal containing $\mathcal{M}$.

Proof. Assume that $\mathcal{M}$ is closed and injective. Given $A$ in ${ }^{a} \mathscr{M}\left(E_{1}, \ldots\right.$, $\left.E_{n} ; F\right)$, Theorem 2.4 yields $i_{F} \circ A \in \overline{M\left(E_{1}, \ldots, E_{n} ; \ell_{\infty}\left(B_{F^{*}}\right)\right)}$. But $\mathcal{M}$ is closed, so $i_{F} \circ A \in \mathscr{M}\left(E_{1}, \ldots, E_{n} ; \ell_{\infty}\left(B_{F^{*}}\right)\right)$. From the injectivity of $\mathcal{M}$ it follows that $A \in \mathscr{M}\left(E_{1}, \ldots, E_{n} ; F\right)$, so $\mathcal{M}={ }^{a} \mathcal{M}$. The converse follows from the fact that $a \mathcal{M}$ is closed and injective (Proposition 2.3). Concerning the last assertion, we know that ${ }^{a} \mathcal{M}$ is a closed injective $n$-ideal containing $\mathscr{M}$. Let $\mathcal{N}$ be a closed injective $n$-ideal containing $\mathscr{M}$. Then, ${ }^{a} \mathscr{M} \subseteq{ }^{a} \mathcal{N}=\mathscr{N}$ by the first assertion.

We thus have that $\mathscr{M} \neq{ }^{a} \mathscr{M}$ if $\mathscr{M}$ fails either to be closed or to be injective. Concrete nonlinear examples will be given in both the closed non-injective case (Example 3.5) and the injective non-closed case (Example 4.2).

\section{Finite type and compact mappings}

In the linear case, the closed injective hull of the ideal $\mathscr{F}$ of finite rank operators coincides with the ideal $\mathscr{K}$ of compact operators, that is ${ }^{a} \mathscr{F}=\mathscr{K}$ (see [21, Proposition 19.2.3]). Denoting by $\mathscr{L}_{\mathscr{K}}$ the closed multi-ideal of compact multilinear mappings (bounded sets are sent onto relatively compact sets), it is obvious to ask if the equality ${ }^{a}\left(\mathscr{L}_{f}\right)=\mathscr{L}_{\mathscr{K}}$ holds true. We begin this section by giving a negative answer.

ExAmple 3.1. Let $A \in \mathscr{L}\left({ }^{2} \ell_{2}\right)$ be given by $A\left(\left(x_{j}\right)_{j=1}^{\infty},\left(y_{j}\right)_{j=1}^{\infty}\right)=$ $\sum_{j=1}^{\infty} x_{j} y_{j}$. Considering the canonical unit vectors we see that $A$ is not weakly sequentially continuous, hence $A$ is not approximable. As $\overline{\mathscr{L}_{f}}$ is closed, by Corollary 2.6 we find that $A \notin{ }^{a}\left(\overline{\mathscr{L}_{f}}\right)\left({ }^{2} \ell_{2}\right)$, hence $A \notin^{a}\left(\mathscr{L}_{f}\right)\left({ }^{2} \ell_{2}\right)$. On the other hand, it is obvious that $A \in \mathscr{L}_{\mathscr{K}}\left({ }^{2} \ell_{2}\right)$.

Once we know that ${ }^{a}\left(\mathscr{L}_{f}\right) \neq \mathscr{L}_{\mathscr{K}}$, it is natural to look for another multiideal which generalizes the compact operators and coincides with ${ }^{a}\left(\mathscr{L}_{f}\right)$. We will accomplish this task almost entirely. First we need some terminology.

The notation $E_{1}, . \hat{i} ., E_{n}$ means that $E_{i}$ is omitted, that is: $\left(E_{1}, . \hat{i} . ., E_{n}\right)=$ $\left(E_{1}, \ldots, E_{i-1}, E_{i+1}, \ldots E_{n}\right)$, the same for $\left(x_{1}, \hat{i} ., x_{n}\right)$. For $i=1, \ldots, n$, consider the isometric isomorphism $I_{i}: \mathscr{L}\left(E_{1}, \ldots, E_{n} ; F\right) \longrightarrow \mathscr{L}\left(E_{i} ; \mathscr{L}\left(E_{1}, \hat{i}\right.\right.$. ,$\left.\left.E_{n} ; F\right)\right)$,

$$
I_{i}(A)\left(x_{i}\right)\left(x_{1}, . \hat{i} ., x_{n}\right)=A\left(x_{1}, \ldots, x_{n}\right) .
$$


For the case $n=1$ to make sense, we consider $I_{1}(A)=A$ for $A \in \mathscr{L}(E ; F)$. Given Banach operator ideals $\mathscr{I}_{1}, \ldots, \mathscr{I}_{n}$, an $n$-linear mapping $A \in \mathscr{L}\left(E_{1}\right.$, $\left.\ldots, E_{n} ; F\right)$ is said to be

- of type $\left[\mathscr{I}_{1}, \ldots, \mathscr{I}_{n}\right]$, and in this case we write $A \in\left[\mathscr{I}_{1}, \ldots, \mathscr{I}_{n}\right]\left(E_{1}, \ldots\right.$, $\left.E_{n} ; F\right)$, if $I_{i}(A) \in \mathscr{I}_{i}\left(E_{i} ; \mathscr{L}\left(E_{1}, . \hat{i} ., E_{n} ; F\right)\right)$, for every $i=1, \ldots, n$.

- of type $\mathscr{L}\left(\mathscr{I}_{1}, \ldots, \mathscr{I}_{n}\right)$, and in this case we write $A \in \mathscr{L}\left(\mathscr{I}_{1}, \ldots, \mathscr{I}_{n}\right)\left(E_{1}\right.$, $\left.\ldots, E_{n} ; F\right)$, if there are Banach spaces $G_{1}, \ldots, G_{n}$, linear operators $u_{j} \in$ $\mathscr{I}_{j}\left(E_{j} ; G_{j}\right), j=1, \ldots, n$, and $B \in \mathscr{L}\left(G_{1}, \ldots, G_{n} ; F\right)$ such that $A=$ $B \circ\left(u_{1}, \ldots, u_{n}\right)$.

If $\mathscr{I}_{1}=\ldots=\mathscr{I}_{n}=\mathscr{I}$ we simply write $[\mathscr{I}]$ and $\mathscr{L}(\mathscr{I})$.

It is well known that $\left[\mathscr{I}_{1}, \ldots, \mathscr{I}_{n}\right]$ and $\mathscr{L}\left(\mathscr{I}_{1}, \ldots, \mathscr{I}_{n}\right)$ are (closed, if $\mathscr{I}_{1}, \ldots$, $\mathscr{I}_{n}$ are closed) $n$-ideals (see [6]). It is clear that $\mathscr{L}\left(\mathscr{I}_{1}, \ldots, \mathscr{I}_{n}\right) \subseteq\left[\mathscr{I}_{1}, \ldots, \mathscr{I}_{n}\right]$. If $\mathscr{I}_{1}, \ldots, \mathscr{I}_{n}$ are closed and injective, then $\mathscr{L}\left(\mathscr{I}_{1}, \ldots, \mathscr{I}_{n}\right)=\left[\mathscr{I}_{1}, \ldots, \mathscr{I}_{n}\right]$ ([10], [20]). In particular, $\mathscr{L}(\mathscr{K})=[\mathscr{K}]$.

Our next aim is to show that ${ }^{a}\left(\mathscr{L}_{f}\right)=[\mathscr{K}]$ modulo the approximation property. Recall that $[\mathscr{K}]$ coincides with the class of multilinear mappings which are weakly continuous on bounded sets [3].

Lemma 3.2. Let $\mathscr{I}, \ldots, \mathscr{I}_{n}$ be operator ideals. If each $\mathscr{I}_{j}$ is injective, then so are $\mathscr{L}\left(\mathscr{I}_{1}, \ldots, \mathscr{I}_{n}\right)$ and $\left[\mathscr{I}_{1}, \ldots, \mathscr{I}_{n}\right]$.

Proof. Let $A \in \mathscr{L}\left(E_{1}, \ldots, E_{n} ; F\right)$ and $i: F \longrightarrow G$ be an isometric embedding. If $i \circ A \in \mathscr{L}\left(\mathscr{I}_{1}, \ldots, \mathscr{I}_{n}\right)\left(E_{1}, \ldots, E_{n} ; G\right)$, we can find $G_{1}, \ldots, G_{n}$, $u_{j} \in \mathscr{I}_{j}\left(E_{j} ; G_{j}\right), j=1, \ldots, n$, and $B \in \mathscr{L}\left(G_{1}, \ldots, G_{n} ; G\right)$ such that $i \circ A=B \circ\left(u_{1}, \ldots, u_{n}\right)$. For $j=1, \ldots, n$, define $u_{j}^{R}: E_{j} \longrightarrow \overline{\operatorname{Range}\left(u_{j}\right)}$ by $u_{j}^{R}\left(x_{j}\right)=u_{j}\left(x_{j}\right)$, and let $i_{j}: \overline{\operatorname{Range}\left(u_{j}\right)} \longrightarrow G_{j}$ be the formal inclusion. Thus $i_{j} \circ u_{j}^{R}=u_{j}$ belongs to $\mathscr{I}_{j}$. The injectivity of $\mathscr{I}_{j}$ yields that each $u_{j}^{R}$ belongs to $\mathscr{I}_{j}$. Define $C \in \mathscr{L}\left(\operatorname{Range}\left(u_{1}\right), \ldots, \operatorname{Range}\left(u_{n}\right) ; F\right)$ by $C\left(u_{1}(x), \ldots, u_{n}(x)\right)=A\left(x_{1}, \ldots, x_{n}\right)$ and extend it continuously to a $\bar{C} \in$ $\mathscr{L}\left(\overline{\operatorname{Range}\left(u_{1}\right)}, \ldots, \overline{\operatorname{Range}\left(u_{n}\right)} ; F\right)$. So $A=\bar{C} \circ\left(u_{1}^{R}, \ldots, u_{n}^{R}\right)$, which shows that $A \in \mathscr{L}\left(\mathscr{I}_{1}, \ldots, \mathscr{I}_{n}\right)\left(E_{1}, \ldots, E_{n} ; F\right)$.

Suppose now that $i \circ A \in\left[\mathscr{I}_{1}, \ldots, \mathscr{I}_{n}\right]\left(E_{1}, \ldots, E_{n} ; G\right)$. Let $j \in\{1, \ldots, n\}$. We know that $I_{j}(i \circ A) \in \mathscr{I}_{j}\left(E_{j} ; \mathscr{L}\left(E_{1}, . . \hat{j} ., E_{n} ; G\right)\right)$. Defining

$$
J_{j}: \mathscr{L}\left(E_{1}, . \hat{j} ., E_{n} ; F\right) \longrightarrow \mathscr{L}\left(E_{1}, \hat{j} . ., E_{n} ; G\right), \quad J_{j}(B)=i \circ B,
$$

it is clear that $J$ is an isometric embedding. For $x_{j} \in E_{j}$ and $\left(x_{1}, . \hat{i} ., x_{n}\right) \in$ 
$E_{1} \times \stackrel{\hat{i}}{\cdots} \times E_{n}$, we have

$$
\begin{aligned}
{\left[\left(J_{j} \circ I_{j}(A)\right)\left(x_{j}\right)\right]\left(x_{1}, . \hat{i} ., x_{n}\right) } & =\left[J_{j}\left(I_{j}(A)\left(x_{j}\right)\right)\right]\left(x_{1}, \hat{i} ., x_{n}\right) \\
& =\left[i \circ\left(I_{j}(A)\left(x_{j}\right)\right)\right]\left(x_{1}, . \hat{i} ., x_{n}\right) \\
& =i\left(\left(I_{j}(A)\left(x_{j}\right)\right)\left(x_{1}, . \hat{i} ., x_{n}\right)\right) \\
& =i\left(A\left(x_{1}, \ldots, x_{n}\right)\right) \\
& =(i \circ A)\left(x_{1}, \ldots, x_{n}\right) \\
& =\left[\left(I_{j}(i \circ A)\right)\left(x_{j}\right)\right]\left(x_{1}, . . \hat{i} ., x_{n}\right) .
\end{aligned}
$$

This shows that $J_{j} \circ I_{j}(A)=I_{j}(i \circ A)$. We have $\mathscr{I}_{j}$ injective, $J_{j} \circ I_{j}(A) \in \mathscr{I}_{j}$ and $J_{j}$ is an isometric embedding. It follows that $I_{j}(A)$ belongs to $\mathscr{I}_{j}$, proving that $A \in\left[\mathscr{I}_{1}, \ldots, \mathscr{I}_{n}\right]\left(E_{1}, \ldots, E_{n} ; F\right)$.

THEOREM 3.3. Suppose that $E_{1}^{*}, \ldots, E_{n}^{*}$ have the approximation property. Then $\left.^{a}\left(\mathscr{L}_{f}\right)\left(E_{1}, \ldots, E_{n} ; F\right)\right)=[\mathscr{K}]\left(E_{1}, \ldots, E_{n} ; F\right)$.

Proof. It is well known that $[\mathscr{K}]$ is a closed (because $\mathscr{K}$ is closed) multiideal. It is injective by Lemma 3.2 as $\mathscr{K}$ is injective, hence $[\mathscr{K}]={ }^{a}[\mathscr{K}]$ by Theorem 2.7. From $\mathscr{L}_{f} \subseteq[\mathscr{K}]$ we conclude that ${ }^{a}\left(\mathscr{L}_{f}\right) \subseteq{ }^{a}[\mathscr{K}]=[\mathscr{K}]$. As mentioned earlier, [ $\mathscr{K}]$ coincides with the class of multilinear mappings which are weakly continuous on bounded sets [3, Theorem 2.9]. Supposing that $E_{1}^{*}, \ldots, E_{n}^{*}$ have the approximation property, [3, Corollary 2.11] gives $[\mathscr{K}]\left(E_{1}, \ldots, E_{n} ; F\right)=\overline{\mathscr{L}_{f}}\left(E_{1}, \ldots, E_{n} ; F\right)$. The proof is complete as $\overline{\mathscr{L}_{f}} \subseteq$ ${ }^{a}\left(\mathscr{L}_{f}\right)$.

The next example shows that Theorem 3.3 does not hold true without the approximation property.

EXAMPLE 3.4. Examining the proof of [2, Theorem 4.5] we find a Banach space $E$ lacking the approximation property and a compact symmetric nonapproximable linear operator $u: E \longrightarrow E^{*}$. Defining $A \in \mathscr{L}\left({ }^{2} E\right)$ by $A(x, y)=u(x)(y)$ it is immediate that $A \in[\mathscr{K}]\left({ }^{2} E\right)$ as $I_{1}(A)=I_{2}(A)=u$. Suppose that $A \in \overline{\mathscr{L}_{f}}\left({ }^{2} E\right)$. Given $\varepsilon>0$, there is $B \in \mathscr{L}\left({ }^{2} E\right)$ of finite type such that $\|A-B\|<\varepsilon$. Say $B=\sum_{j=1}^{k} \varphi_{i} \psi_{i}, \varphi_{1}, \ldots, \varphi_{k}, \psi_{1}, \ldots, \psi_{k} \in E^{*}$. Defining $v \in \mathscr{L}\left(E ; E^{*}\right)$ by $v(x)=\sum_{j=1}^{k} \varphi_{i}(x) \psi_{i}$ we have that $v$ is a finite rank operator. Furthermore,

$\|u(x)(y)-v(x)(y)\|=\|A(x, y)-B(x, y)\| \leq\|A-B\|\|x\|\|y\|<\varepsilon\|x\|\|y\|$,

for every $x, y \in E$. It results that $\|u-v\| \leq \varepsilon$, which shows that $u$ is approximable, a contradiction. So, $A \notin \overline{\mathscr{L}_{f}}\left({ }^{2} E\right)$, and by Corollary 2.6 we have $A \notin{ }^{a}\left(\mathscr{L}_{f}\right)\left({ }^{2} E\right)$. 
Thus far we know that ${ }^{a}\left(\mathscr{L}_{f}\right)=\overline{\mathscr{L}_{f}}$ if either the range space is injective (Corollary 2.6) or the duals of the domain spaces have the approximation property (Theorem 3.3). In the linear case, we have already mentioned that $a \mathscr{F}=\mathscr{K}$, so any compact non-approximable linear operator assures that $a \mathscr{F} \neq$ $\overline{\mathscr{F}}$. Let us see a nonlinear example.

ExAmple 3.5. Let $u: E \longrightarrow F$ be a compact non-approximable linear operator, for example the operator from Example 3.4 (actually, for every Banach space $E$ without the approximation property there is a Banach space $F$ and a compact non-approximable operator $u$ from $E$ to $F$ ). Fix $\varphi \in E^{*},\|\varphi\|=1$, and $a \in E$ with $\varphi(a)=1$. Define

$$
A: E \times E \longrightarrow F, \quad A(x, y)=\varphi(x) u(y) .
$$

Suppose that $A$ belongs to $\overline{\mathscr{L}_{f}}$. Given $\varepsilon>0$, there are $\varphi_{1}, \ldots, \varphi_{k}, \psi_{1}, \ldots$, $\psi_{k} \in E^{*}$ and $b, \ldots, b_{k} \in F$ such that $\left\|A-\sum_{j=1}^{k} \varphi_{j} \psi_{j} b_{j}\right\|<\frac{\varepsilon}{\|a\|}$. For every $y \in E$ we have

$$
\left\|u(y)-\sum_{j=1}^{k} \varphi_{j}(a) \psi_{j}(y) b_{j}\right\|=\left\|A(a, y)-\sum_{j=1}^{k} \varphi_{j}(a) \psi_{j}(y) b_{j}\right\|<\frac{\varepsilon}{\|a\|}\|a\|\|y\|,
$$

resulting $\left\|u-\sum_{j=1}^{k} \varphi_{j}(a) \psi_{j} b_{j}\right\| \leq \varepsilon$. But $\sum_{j=1}^{k} \varphi_{j}(a) \psi_{j} b_{j}$ is a finite rank operator, so $u$ is approximable, a contradiction. Hence $A \notin \overline{\mathscr{L}_{f}}\left({ }^{2} E ; F\right)$. In order to show that $A \in{ }^{a}\left(\mathscr{L}_{f}\right)\left({ }^{2} E ; F\right)$, let $\varepsilon>0$. Since $u$ is compact, by [21, Proposition 19.2.3] there is a finite rank operator $v: E \longrightarrow \ell_{\infty}\left(B_{F^{*}}\right)$ such that $\left\|i_{F} \circ u-v\right\|<\frac{\varepsilon}{\|\varphi\|}$. Defining $B \in \mathscr{L}\left({ }^{2} E ; \ell_{\infty}\left(B_{F^{*}}\right)\right)$ by $B(x, y)=\varphi(x) v(y)$ we have that $B$ is of finite type and

$$
\begin{aligned}
\left\|i_{F} \circ A(x, y)-B(x, y)\right\| & =\left\|i_{F}(\varphi(x) u(y))-\varphi(x) v(y)\right\| \\
& =\mid \varphi(x)\|\| i_{F}(u(y))-v(y) \| \\
& \leq\|\varphi\|\left\|i_{F} \circ u-v\right\|\|x\|\|y\| \\
& <\varepsilon\|x\|\|y\|,
\end{aligned}
$$

for every $x, y \in E$. It follows that $\left\|i_{F} \circ A-B\right\| \leq \varepsilon$, so by Theorem 2.4 we have that $A \in{ }^{a}\left(\mathscr{L}_{f}\right)\left({ }^{2} E ; F\right)$.

We have seen that, contrary to the linear case, $[\mathscr{K}] \nsubseteq^{a}\left(\mathscr{L}_{f}\right)$. Next we show that this is caused by the fact that multilinear forms are not always of finite type. By $\mathscr{L}_{\phi}\left(E_{1}, \ldots, E_{n} ; F\right)$ we denote the subspace of $\mathscr{L}\left(E_{1}, \ldots, E_{n} ; F\right)$ spanned by the mappings of the form $A\left(x_{1}, \ldots, x_{n}\right)=B\left(x_{1}, \ldots, x_{n}\right) b$ where $B \in \mathscr{L}\left(E_{1}, \ldots, E_{n}\right)$ and $b \in F$. 
Proposition 3.6. If $A \in[\mathscr{K}]\left(E_{1}, \ldots, E_{n} ; F\right)$, then for every $\varepsilon>0$ there is an n-linear mapping $C \in \mathscr{L}_{\phi}\left(E_{1}, \ldots, E_{n} ; \ell_{\infty}\left(B_{F^{*}}\right)\right)$ such that $\left\|i_{F} \circ A-C\right\|<$ $\varepsilon$.

Proof. Since $\mathscr{L}(\mathscr{K})=[\mathscr{K}]$, let $G_{1}, \ldots, G_{n}, u_{j} \in \mathscr{K}\left(E_{j} ; G_{j}\right)$ and $B \in$ $\mathscr{L}\left(G_{1}, \ldots, G_{n} ; F\right)$ be such that $A=B \circ\left(u_{1}, \ldots, u_{n}\right)$. It follows that $u_{1} \otimes \cdots \otimes$ $u_{n}$ is a compact operator from $E_{1} \widehat{\bigotimes}_{\pi} \cdots \widehat{\otimes}_{\pi} E_{n}$ to $G_{1} \widehat{\bigotimes}_{\pi} \cdots \widehat{\bigotimes}_{\pi} G_{n}$ (see [22] 44.6.1). So, $i_{F} \circ B_{L} \circ u_{1} \otimes \cdots \otimes u_{n}$ is a compact operator from $E_{1} \widehat{\otimes}_{\pi} \cdots \widehat{\otimes}_{\pi} E_{n}$ to $\ell_{\infty}\left(B_{F^{*}}\right)$. The latter space has the approximation property, hence there are $\varphi_{1}, \ldots, \varphi_{k} \in\left(E_{1} \widehat{\otimes}_{\pi} \ldots \widehat{\otimes}_{\pi} E_{n}\right)^{*}$ and $b_{1}, \ldots, b_{k} \in \ell_{\infty}\left(B_{F^{*}}\right)$ such that

$$
\left\|i_{F} \circ B_{L} \circ u_{1} \otimes \cdots \otimes u_{n}-\sum_{j=1}^{k} \varphi_{j} b_{j}\right\|<\varepsilon .
$$

For $j=1, \ldots, k$, take $B_{j} \in \mathscr{L}\left(E_{1}, \ldots, E_{n}\right)$ such that $\left(B_{j}\right)_{L}=\varphi_{j}$. For $\left(x_{1}, \ldots, x_{n}\right) \in E_{1} \times \cdots \times E_{n}$ it is clear that $B_{L} \circ u_{1} \otimes \cdots \otimes u_{n}\left(x_{1} \otimes \cdots \otimes x_{n}\right)=$ $B\left(u_{1}\left(x_{1}\right), \ldots, u_{n}\left(x_{n}\right)=A\left(x_{1}, \ldots, x_{n}\right)\right.$, so $\left\|i_{F} \circ A-\sum_{j=1}^{k} B_{j} b_{j}\right\|<\varepsilon$.

\section{Weakly compact and absolutely summing mappings}

By $\mathscr{W}$ and $\mathscr{L}_{\mathscr{W}}$ we mean the ideals of weakly compact linear operators and multilinear mappings respectively (bounded sets are sent onto relatively weakly compact sets) and by $\Pi_{p}$ the ideal of absolutely $p$-summing linear operators. In this section we investigate multilinear counterparts of properties of ${ }^{a} \Pi_{p}$ and their connections with $\mathscr{W}$. The ideal $\Pi_{p}$ has been generalized to the multilinear setting in several ways, and among the most studied ones we find the multiideal of dominated mappings:

Definition 4.1. Let $p_{1}, \ldots, p_{n} \geq 1$. An $n$-linear mapping $A \in \mathscr{L}\left(E_{1}, \ldots\right.$, $\left.E_{n} ; F\right)$ is $\left(p_{1}, \ldots, p_{n}\right)$-dominated if there is a constant $C \geq 0$ such that

$$
\left(\sum_{j=1}^{k}\left\|A\left(x_{j}^{1}, \ldots, x_{j}^{n}\right)\right\|^{r}\right)^{\frac{1}{r}} \leq C \prod_{i=1}^{n} \sup _{\varphi \in B_{E_{i}^{*}}^{*}}\left(\sum_{j=1}^{k}\left|\varphi\left(x_{j}^{i}\right)\right|^{p_{i}}\right)^{\frac{1}{p_{i}}}
$$

where $\frac{1}{r}=\frac{1}{p_{1}}+\cdots+\frac{1}{p_{n}}$, for every $k \in \mathrm{N}$ and any $x_{j}^{i} \in E_{i}, j=1, \ldots, k, i=$ $1, \ldots, n$. In this case we write $A \in \mathscr{L}_{d ; p_{1}, \ldots, p_{n}}\left(E_{1}, \ldots, E_{n} ; F\right)$. Denoting the infimum of the constants $C$ working in the inequality by $\|A\|_{d ; p_{1}, \ldots, p_{n}}$ we have that $\left(\mathscr{L}_{d ; p_{1}, \ldots, p_{n}},\|\cdot\|_{d ; p_{1}, \ldots, p_{n}}\right)$ is a complete $r$-normed $n$-ideal. If $p_{1}=\cdots=$ $p_{n}=p$ we say that $A$ is $p$-dominated and write $A \in \mathscr{L}_{d ; p}\left(E_{1}, \ldots, E_{n} ; F\right)$.

Before going into the main results of the section we provide the announced nonlinear examples of mappings in ${ }^{a} \mathcal{M}$ but not in $\mathscr{M}$ for injective non-closed 
$\mathcal{M}$. The characterization

$$
\mathscr{L}_{d ; p_{1}, \ldots, p_{n}}=\mathscr{L}\left(\Pi_{p_{1}}, \ldots, \Pi_{p_{n}}\right),
$$

which goes back to [25] (a detailed proof can be found in [23, Corolario 3.23]), shall be useful several times.

EXAMPLE 4.2. Since multilinear forms on $c_{0}$ are approximable we have that $\mathscr{L}\left({ }^{n} c_{0}\right)={ }^{a}\left(\mathscr{L}_{d, p}\right)\left({ }^{n} c_{0}\right)$ for every $p \geq n$. On the other hand, $\mathscr{L}\left({ }^{n} c_{0}\right) \neq$ $\mathscr{L}_{d, p}\left({ }^{n} c_{0}\right)$ for $n \geq 3$ and $p \geq 1$ by [4, Theorem 3.5]. So $\mathscr{L}_{d, p}\left({ }^{n} c_{0}\right) \neq$ ${ }^{a}\left(\mathscr{L}_{d, p}\right)\left({ }^{n} c_{0}\right)$ for $p \geq n \geq 3$. Of course this is an implicit example. Let us see an explicit one: let $u \in \mathscr{L}(E ; F)$ be a 3-summing non-2-summing linear operator (for example the canonical map $j_{3}: C[0,1] \longrightarrow L_{3}[0,1]$ ). Fix $\varphi \in E^{*},\|\varphi\|=1$, and define $A \in \mathscr{L}\left({ }^{2} E ; F\right)$ by $A(x, y)=\varphi(x) u(y)$. Since $u$ is 3-summing, $u$ is absolutely continuous, so by [14, page 311] there are a Banach space $G$ and a 2-summing operator $j: E \longrightarrow G$ such that for every $\varepsilon>0$ there is $K_{\varepsilon}$ such that

$$
\|u(x)\| \leq K_{\varepsilon}\|j(x)\|+\varepsilon\|x\| \quad \text { for every } \quad x \in E .
$$

Define $B \in \mathscr{L}\left({ }^{2} E ; G\right)$ by $B(x, y)=\varphi(x) j(y)$. Since $B=C \circ(\varphi, j)$, where $C(\lambda, y)=\lambda y$, it follows from $(*)$ that $B$ is 2-dominated. From

$$
\begin{aligned}
\left\|\sum_{i=1}^{k} A\left(x_{i}, y_{i}\right)\right\| & =\left\|u\left(\sum_{i=1}^{k} \varphi\left(x_{i}\right) y_{i}\right)\right\| \\
& \leq K_{\varepsilon}\left\|j\left(\sum_{i=1}^{k} \varphi\left(x_{i}\right) y_{i}\right)\right\|+\varepsilon\left\|\sum_{i=1}^{k} \varphi\left(x_{i}\right) y_{i}\right\| \\
& \leq K_{\varepsilon}\left\|\sum_{i=1}^{k} B\left(x_{i}, y_{i}\right)\right\|+\varepsilon \sum_{i=1}^{k}\left\|x_{i}\right\|\left\|y_{i}\right\|,
\end{aligned}
$$

we conclude that $A \in{ }^{a}\left(\mathscr{L}_{d, 2}\right)\left({ }^{2} E ; F\right)$. Suppose that $A$ is 2-dominated. By (*) $A$ can be written as $A=C \circ\left(v_{1}, v_{2}\right)$ with $v_{1}, v_{2}$ being 2 -summing. Choosing $a \in E$ with $\varphi(a)=1$, for every $x \in E$,

$$
u(x)=\varphi(a) u(x)=A(a, x)=C\left(v_{1}(a), v_{2}(x)\right)=\left(C \circ\left(v_{1}(a), \cdot\right) \circ v_{2}\right)(x),
$$

resulting $u=\left(C \circ\left(v_{1}(a), \cdot\right)\right) \circ v_{2}$. This is absurd because $v_{2}$ is 2-summing whereas $u$ is not, so $A$ fails to be 2-dominated.

Recall that ${ }^{a} \Pi_{p}=\mathscr{H}$, the ideal of absolutely continuous operators. Since every absolutely continuous operator is weakly compact and completely continuous [14, Corollary 15.4], it is natural to wonder whether every multilinear 
mapping belonging to $a \mathscr{L}_{d ; p_{1}, \ldots, p_{n}}$ is (a) weakly compact and/or (b) weakly sequentially continuous (for multilinear mappings the literature speaks of weakly sequentially continuous mappings rather than completely continuous mappings).

Since there are $p$-dominated non-weakly compact $n$-linear mappings [5, Example 1], we have $\mathscr{L}_{d, p} \subseteq{ }^{a} \mathscr{L}_{d, p} \nsubseteq \mathscr{L}_{\mathscr{W}}$, so (a) does not hold in general. This leads us to consider a more suitable standard multilinear generalization of weakly compact operators: the ideal of Arens-regular multilinear mappings $[\mathscr{W}]$.

Proposition 4.3. Every multilinear mapping belonging to ${ }^{a} \mathscr{L}_{d ; p_{1}, \ldots, p_{n}}$ is weakly sequentially continuous and Arens-regular.

Proof. First let us show that dominated multilinear mappings are weakly sequentially continuous. Given $C \in \mathscr{L}_{d ; p_{1}, \ldots, p_{n}}\left(E_{1}, \ldots, E_{n} ; F\right)$, applying $(*)$ once more we can write $C=B \circ\left(u_{1}, \ldots, u_{n}\right)$ where each $u_{j}$ is $p_{j}$-summing. Since $p_{j}$-summing operators are completely continuous and $B$ is continuous, it follows that $C$ is weakly sequentially continuous. Now consider $A \in$ $a \mathscr{L}_{d ; p_{1}, \ldots, p_{n}}\left(E_{1}, \ldots, E_{n} ; F\right)$. By Theorem 2.4,

$$
i_{F} \circ A \in \overline{\mathscr{L}_{d ; p_{1}, \ldots, p_{n}}\left(E_{1}, \ldots, E_{n} ; \ell_{\infty}\left(B_{F^{*}}\right)\right)} .
$$

Moreover the space of weakly sequentially continuous $n$-linear mappings from $E_{1} \times \cdots \times E_{n}$ to $\ell_{\infty}\left(B_{F^{*}}\right)$ is closed and by the first part of the proof it contains the $\left(p_{1}, \ldots, p_{n}\right)$-dominated mappings, so $i_{F} \circ A$ is weakly sequentially continuous. As $i_{F}$ is an isometric embedding, it follows that $A$ is weakly sequentially continuous.

Recall that $p_{j}$-summing operators are weakly compact. Thus $\left[\Pi_{p_{1}}, \ldots\right.$, $\left.\Pi_{p_{n}}\right] \subseteq[\mathscr{W}]$. Since $[\mathscr{W}]$ is closed and injective and $\mathscr{L}\left(\Pi_{p_{1}}, \ldots, \Pi_{p_{n}}\right) \subseteq$ $\left[\Pi_{p_{1}}, \ldots, \Pi_{p_{n}}\right]$, the second assertion follows from Theorem 2.7.

Remark 4.4. Although there are Banach spaces $F$ such that $\mathscr{L}\left(\ell_{1} ; F\right)=$ ${ }^{a} \Pi_{p}\left(\ell_{1} ; F\right)$, like Hilbert spaces or reflexive quotients of a $C(K)$ space [14, p. 313], such type of coincidence does not hold for multilinear mappings, that is $\mathscr{L}\left({ }^{n} \ell_{1} ; F\right) \neq{ }^{a} \mathscr{L}_{d ; p_{1}, \ldots, p_{n}}\left({ }^{n} \ell_{1} ; F\right)$ for $n \geq 2, p_{1}, \ldots, p_{n} \geq 1$ and all Banach spaces $F$. Indeed, were the equality true for some $n, p_{1}, \ldots, p_{n}$ and some $F$, combining $(*)$ with [6, Proposition 41] we would have $\mathscr{L}\left({ }^{2} \ell_{1} ; F\right)=$ ${ }^{a} \mathscr{L}_{d ; p_{1}, p_{2}}\left({ }^{2} \ell_{1} ; F\right)$. So the same equality would hold true for any complemented subspace of $F$, thus for the scalar field. However $\mathscr{L}\left({ }^{2} \ell_{1}\right) \neq a \mathscr{L}_{d ; p_{1}, p_{2}}\left({ }^{2} \ell_{1}\right)$ since the bilinear form on $\ell_{1}$ constructed in [1, p. 83] is not Arens-regular.

As mentioned earlier, ${ }^{a} \Pi_{p}=\mathscr{H}$, so it follows that ${ }^{a} \Pi_{p}={ }^{a} \Pi_{q}$ for every $p, q \geq 1$ (see also [21, Corollary 20.7.7]). Although we do not know whether 
${ }^{a} \mathscr{L}_{d ; p_{1}, \ldots, p_{n}}={ }^{a} \mathscr{L}_{d ; q_{1}, \ldots, q_{n}}$, we are able to identify another multilinear generalization of the ideal of absolutely summing linear operators in which this phenomenon does occur (as usual, the properties of a given operator ideal are to be found among its several multilinear generalizations, rather than in a specific one):

Definition 4.5 (Composition ideals - see [8], [16]). Let $\mathscr{I}$ be a Banach operator ideal. An $n$-linear mapping $A \in \mathscr{L}\left(E_{1}, \ldots, E_{n} ; F\right)$ belongs to $\mathscr{I} \circ \mathscr{L}$ -in this case we write $A \in \mathscr{I} \circ \mathscr{L}\left(E_{1}, \ldots, E_{n} ; F\right)$-if there are a Banach space $G$, an $n$-linear mapping $B \in \mathscr{L}\left(E_{1}, \ldots, E_{n} ; G\right)$ and an operator $u \in \mathscr{I}(G ; F)$ such that $A=u \circ B . \mathscr{I} \circ \mathscr{L}$ is a multi-ideal which becomes a Banach multi-ideal with the norm

$$
\begin{aligned}
& \|A\|_{\mathscr{I} O \mathscr{L}} \\
& \quad:=\inf \left\{\|u\|_{\mathscr{I}}\|B\|: A=u \circ B, B \in \mathscr{L}\left(E_{1}, \ldots, E_{n} ; G\right), u \in \mathscr{I}(G ; F)\right\} .
\end{aligned}
$$

Proposition 4.6. ${ }^{a}(\mathscr{I} \circ \mathscr{L})={ }^{a} \mathscr{I} \circ \mathscr{L}$ for every Banach operator ideal $\mathscr{I}$. In particular, ${ }^{a}\left(\Pi_{p} \circ \mathscr{L}\right)={ }^{a}\left(\Pi_{q} \circ \mathscr{L}\right)$ for every $p, q \geq 1$.

Proof. Assume for a while that $\mathscr{I}$ is injective. Let $A \in \mathscr{L}\left(E_{1}, \ldots, E_{n} ; F\right)$ and $i: F \rightarrow G$ be an isometric embedding such that $i \circ A \in \mathscr{I} \circ \mathscr{L}\left(E_{1}, \ldots, E_{n} ; G\right)$. Notice that according to [8, Proposition 2.2], $(i \circ A)_{L} \in \mathscr{I}\left(E_{1} \widehat{\otimes}_{\pi} \cdots \widehat{\otimes}_{\pi} E_{n} ; G\right)$. Since $i \circ A_{L}=(i \circ A)_{L}$, it follows that $i \circ A_{L} \in \mathscr{I}\left(E_{1} \widehat{\otimes}_{\pi} \cdots \widehat{\otimes}_{\pi} E_{n} ; G\right)$. The injectivity of $\mathscr{I}$ gives $A_{L} \in \mathscr{I}\left(E_{1} \widehat{\otimes}_{\pi} \cdots \widehat{\otimes}_{\pi} E_{n} ; F\right)$, so the factorization $A=A_{L} \circ \sigma_{n}$, where $\sigma_{n}: E_{1} \times \cdots \times E_{n} \longrightarrow E_{1} \widehat{\otimes}_{\pi} \cdots \widehat{\otimes}_{\pi} E_{n}$ is the canonical $n$-linear mapping given by $\sigma_{n}\left(x_{1}, \ldots, x_{n}\right)=x_{1} \otimes \cdots \otimes x_{n}$, shows that $A \in$ $\mathscr{I} \circ \mathscr{L}\left(E_{1}, \ldots, E_{n} ; F\right)$. Thus far we have proved that $\mathscr{I} \circ \mathscr{L}$ is injective whenever $\mathscr{I}$ is injective. As ${ }^{a} \mathscr{I}$ is closed and injective, ${ }^{a} \mathscr{I} \circ \mathscr{L}$ is closed and injective as well, so ${ }^{a} \mathscr{I} \circ \mathscr{L}={ }^{a}\left({ }^{a} \circ \mathscr{L}\right)$. From $\mathscr{I} \subseteq{ }^{a} \mathscr{I}$ we get $\mathscr{I} \circ \mathscr{L} \subseteq{ }^{a} \mathscr{I} \circ \mathscr{L}$, hence ${ }^{a}(\mathscr{I} \circ \mathscr{L}) \subseteq{ }^{a}\left({ }^{a} \mathscr{I} \circ \mathscr{L}\right)={ }^{a} \mathscr{I} \circ \mathscr{L}$.

Given $A \in \mathscr{I}^{a} \circ \mathscr{L}\left(E_{1}, \ldots, E_{n} ; F\right)$, write $A=u \circ B$ with $B \in \mathscr{L}\left(E_{1}, \ldots\right.$, $\left.E_{n} ; G\right)$ and $u \in a \mathscr{I}(G ; F)$. Given $\varepsilon>0$, there exists an operator $v \in$ $\mathscr{I}\left(G ; \ell_{\infty}\left(B_{F^{*}}\right)\right)$ such that $\left\|v-i_{F} \circ u\right\|<\frac{\varepsilon}{\|B\|}$. In this fashion $v \circ B \in$ $\mathscr{I} \circ \mathscr{L}\left(E_{1}, \ldots, E_{n} ; \ell_{\infty}\left(B_{F^{*}}\right)\right)$ and

$$
\left\|v \circ B-i_{F} \circ A\right\|=\left\|v \circ B-i_{F} \circ u \circ B\right\| \leq\left\|v-i_{F} \circ u\right\|\|B\|<\varepsilon .
$$

This shows, by Theorem 2.4, that $A \in{ }^{a}(\mathscr{I} \circ \mathscr{L})\left(E_{1}, \ldots, E_{n} ; F\right)$. 


\section{Aron-Berner extensions}

In this section we prove that the correspondence $\mathscr{M} \mapsto{ }^{a} \mathscr{M}$ preserves AronBerner stability. Recall that the Aron-Berner extension, sometimes called Arens extension or canonical extension, of a multilinear mapping $A \in \mathscr{L}\left(E_{1}\right.$, $\left.\ldots, E_{n} ; F\right)$, denoted $\widetilde{A} \in \mathscr{L}\left(E_{1}^{* *}, \ldots, E_{n}^{* *} ; F^{* *}\right)$, is defined according to $\widetilde{A}\left(x_{1}^{* *}, \ldots, x_{n}^{* *}\right)(\varphi)=\lim _{i_{1}} \ldots \lim _{i_{n}}(\varphi \circ A)\left(x_{i_{1}}, \ldots, x_{i_{n}}\right) \quad$ for all $\varphi \in F^{*}$,

where the limits are iterated limits and $\left(x_{i_{l}}\right)$ is a net in $E_{l}$ weak* converging to $x_{l}^{* *} \in E_{l}^{* *}$. It turns out that $\widetilde{A}\left(x_{1}^{* *}, \ldots, x_{n}^{* *}\right)=\lim _{i_{1}} \ldots \lim _{i_{n}} A\left(x_{i_{1}}, \ldots, x_{i_{n}}\right)$ where the limits are taken in the $w\left(F^{* *}, F^{*}\right)$ topology. The mapping

$$
A \in \mathscr{L}\left(E_{1}, \ldots, E_{n} ; F\right) \mapsto \widetilde{A} \in \mathscr{L}\left(E_{1}^{* *}, \ldots, E_{n}^{* *} ; F^{* *}\right)
$$

is a linear into isometry. See [15] for more information about this bidual extension. Depending on the order the iterated limits are taken, $A$ can have several (at most $n$ !) different Aron-Berner extensions.

Definition 5.1. An $n$-ideal $\mathscr{M}$ is said to be Aron-Berner stable if all AronBerner extensions of any $n$-linear mapping in $\mathscr{M}$ also belong to $\mathcal{M}$.

Some multi-ideals are known to be Aron-Berner stable (for example, integral mappings [12, Proposition 8]) and some are known not to be (for example, weakly sequentially continuous mappings [26, Example 1.9]).

THeOREm 5.2. Let $M$ be an Aron-Berner stable $n$-ideal. The following are equivalent for a multilinear mapping $A \in \mathscr{L}\left(E_{1}, \ldots, E_{n} ; F\right)$ :

(a) $A \in{ }^{a} \mathcal{M}\left(E_{1}, \ldots, E_{n} ; F\right)$.

(b) All Aron-Berner extensions of A belong to ${ }^{a} \mathcal{M}\left(E_{1}^{* *}, \ldots, E_{n}^{* *} ; F^{* *}\right)$.

(c) Some Aron-Berner extension of $A$ belongs to ${ }^{a} \mathcal{M}\left(E_{1}^{* *}, \ldots, E_{n}^{* *} ; F^{* *}\right)$. In particular, ${ }^{a} \mathcal{M}$ is Aron-Berner stable.

Proof. (a) $\Longrightarrow$ (b) Let $\widetilde{A}$ be an Aron-Berner extension of $A$. In what follows the notation $\widetilde{B}$ means the Aron-Berner extension of $B$ where the iterated limits are taken in the same order as in $\widetilde{A}$. Firstly we remark that the natural embedding

$$
I_{F^{* *}}: x^{* *} \in F^{* *} \mapsto\left(\left\langle x^{* *}, \varphi\right\rangle\right)_{\varphi \in B_{F^{*}}} \in \ell_{\infty}\left(B_{F^{*}}\right),
$$

is a linear isometry that extends the isometric embedding $i_{F}$ and it is weak*weak* continuous since it is the transpose of the mapping

$$
\left(a_{\varphi}\right)_{\varphi \in B_{F^{*}}} \in \ell_{1}\left(B_{F^{*}}\right) \mapsto \sum_{\varphi \in B_{F^{*}}} a_{\varphi} \cdot \varphi \in F^{*} .
$$


This remark proves that $i_{F} \circ A=I_{F^{* *} \circ} \widetilde{A}$. Assume that $A \in{ }^{a} \mathcal{M}\left(E_{1}, \ldots, E_{n} ; F\right)$ and fix $\varepsilon>0$. According to condition (b) in Theorem 2.4, there is an $n$-linear mapping $C \in \mathcal{M}\left(E_{1}, \ldots, E_{n} ; \ell_{\infty}\left(B_{F^{*}}\right)\right)$ such that $\left\|i_{F} \circ A-C\right\|<\varepsilon$. Hence

$$
\left\|I_{F^{* *}} \circ \widetilde{A}-\widetilde{C}\right\|=\left\|i_{F} \circ A-\widetilde{C}\right\|=\left\|\left(i_{F} \circ \widetilde{A}-C\right)\right\|<\varepsilon .
$$

The Aron-Berner stability of $\mathcal{M}$ assures that $\widetilde{C}$ belongs to $\mathcal{M}$, thus we have seen that $I_{F^{* *}} \circ \widetilde{A}$ is in the closure of $\mathscr{M}\left(E_{1}^{* *}, \ldots, E_{n}^{* *} ;\left(\ell_{\infty}\left(B_{F^{*}}\right)\right)^{* *}\right)$. Hence $I_{F^{* *}} \circ \widetilde{A} \in{ }^{a} \mathcal{M}\left(E_{1}^{* *}, \ldots, E_{n}^{* *} ;\left(\ell_{\infty}\left(B_{F^{*}}\right)\right)^{* *}\right)$, and because of the injectivity of $\left.{ }^{a} \mathcal{M}, \widetilde{A} \in{ }^{a} M\left(E_{1}^{* *}, \ldots, E_{n}^{* *} ; F^{* *}\right)\right)$.

(b) $\Longrightarrow$ (c) is obvious.

(c) $\Longrightarrow$ (a) By $J_{E}$ we mean the canonical isometric embedding from $E$ into $E^{* *}$. Let $\widetilde{A}$ be an Aron-Berner extension of $A$ belonging to ${ }^{a} \mathcal{M}\left(E_{1}^{* *}, \ldots, E_{n}^{* *}\right.$; $\left.F^{* *}\right)$. The ideal property yields that $J_{F} \circ A=\widetilde{A} \circ\left(J_{E_{1}}, \ldots, J_{E_{n}}\right) \in{ }^{a} \mathcal{M}\left(E_{1}, \ldots\right.$, $\left.E_{n} ; F^{* *}\right)$. From the injectivity of ${ }^{a} \mathcal{M}$ it follows that $A \in{ }^{a} \mathscr{M}\left(E_{1}, \ldots, E_{n} ; F\right)$.

It is well known that a linear operator $u$ belongs to ${ }^{a} \Pi_{p}$, that is, $u$ is absolutely continuous, if and only if $u^{* *}$ belongs to ${ }^{a} \Pi_{p}$ as well [14, Corollary 15.5]. It is clear that Theorem 5.2 generalizes this result to multilinear mapings. Moreover, taking $n=1$ in Theorem 5.2 one sees that even in the linear case [14, Corollary 15.5] is a particular case of a much more general situation:

Corollary 5.3. Let $\mathscr{I}$ be an operator ideal such that $u \in \mathscr{I} \Longrightarrow u^{* *} \in \mathscr{I}$. Given $u \in \mathscr{L}(E ; F), u \in{ }^{a} \mathscr{I}(E ; F)$ if and only if $u^{* *} \in{ }^{a} \mathscr{I}\left(E^{* *} ; F^{* *}\right)$.

For instance, maximal Banach operator ideals satisfy the condition $u \in$ $\mathscr{I} \Longrightarrow u^{* *} \in \mathscr{I}\left[13\right.$, Corollary 17.8.4] (observe that, for being closed, ${ }^{a} \mathscr{I}$ is maximal only if $a \mathscr{I}=\mathscr{L}$ ). Let us stress that the above corollary is a genuine generalization of [14, Corollary 15.5]: indeed, on the one hand the proof of [14, Corollary 15.5] works only for operator ideals contained in $\mathscr{W}$, on the other hand there are maximal operator ideals not contained in $\mathscr{W}$, for example the ideal of cotype 2 operators (cf. [13, 17.4]).

Acknowledgements. Part of this paper was written while G. B. and L. P. were visiting the Departamento de Análisis Matemático at Universidad de Valencia. They thank Pablo Galindo, Pilar Rueda and the members of the department for their hospitality and support.

\section{REFERENCES}

1. Aron, R., Cole, B., and Gamelin, T., Spectra of algebras of analytic functions on a Banach space, J. reine angew. Math. 415 (1991), 51-93.

2. Aron, R., Cole, B., and Gamelin, T., Weak-star continuous analytic functions, Canad. J. Math. 47 (1995), 673-683. 
3. Aron, R., Hervés, C., and Valdivia, M., Weakly continuous mappings on Banach spaces, J. Funct. Anal. 52 (1983), 189-204.

4. Botelho, G., Cotype and absolutely summing multilinear mappings and homogeneous polynomials, Proc. Roy. Irish Acad. Sect. A 97 (1997), 145-153.

5. Botelho, G., Weakly compact and absolutely summing polynomials, J. Math. Anal. Appl. 265 (2002), 458-462.

6. Botelho, G., Ideals of polynomials generated by weakly compact operators, Note Mat. 25 (2005), 69-102.

7. Botelho, G., Braunss, H.-A., Junek, H., and Pellegrino, D., Holomorphy types and ideals of multilinear mappings, Studia Math. 177 (2006), 43-65.

8. Botelho, G., Pellegrino, D., and Rueda, P., On composition ideals of multilinear mappings and homogeneous polynomials, Publ. Res. Inst. Math. Sci. 43 (2007), 1139-1155.

9. Braunss, H.-A., Ideale multilinearer Abbildungen und Räume holomorpher Funktionen, Dissertation (A), Pädagogische Hochschule Karl Liebknecht, Potsdam 1984.

10. Braunss, H.-A., and Junek, H., Factorization of injective ideals by interpolation, J. Math. Anal. Appl. 297 (2004), 740-750.

11. Carando, D., Dimant, V., and Sevilla-Peris, P., Limit orders and multilinear forms on $\ell_{p}$ spaces, Publ. Res. Inst. Math. Sci. 42 (2006), 507-522.

12. Carando, D., and Lassalle, S., Extension of vector-valued integral polynomials, J. Math. Anal. Appl. 307 (2005), 77-85.

13. Defant, A., and Floret, K., Tensor Norms and Operator Ideals, North-Holland Math. Studies 174, North-Holland, Amsterdam 1993.

14. Diestel, J., Jarchow, H., and Tonge, A., Absolutely Summing Operators, Cambridge Studies in Advanced Math. 43, Cambridge Univ. Press, Cambridge 1995.

15. Dineen, S., Complex Analysis on Infinite-Dimensional Spaces, Springer, London 1999.

16. Floret, K., Minimal ideals of n-homogeneous polynomials on Banach spaces, Results Math. 39 (2001), 210-217.

17. Floret, K., On ideals of n-homogeneous polynomials on Banach spaces, pp. 19-38 in: Topological Algebras with Applications to Differential Geometry and Mathematical Physics (Proc. Athens 1999), Univ. Athens, Athens 2002.

18. Floret, K., and Hunfeld, S., Ultrastability of ideals of homogeneous polynomials and multilinear mappings on Banach spaces, Proc. Amer. Math. Soc. 130 (2002), 1425-1435.

19. Floret, K., and García, D., On ideals of polynomials and multilinear mappings between Banach spaces, Arch. Math. (Basel) 81 (2003), 300-308.

20. González, M., and Gutiérrez, J., Injective factorization of holomorphic mappings, Proc. Amer. Math. Soc. 127 (1999), 1715-1721. See also Erratum in vol. 129 (2001), 1255-1256.

21. Jarchow, H., Locally Convex Spaces, Teubner, Stuttgart 1981.

22. Köthe, G., Topological Vector Spaces II, Grundlehren der math. Wissenschaften 237, Springer, New York 1979.

23. Pérez-García, D., Operadores multilineales absolutamente sumantes, Tesina, Universidad Complutense de Madrid, 2002.

24. Pérez-García, D., Comparing different classes of absolutely summing multilinear operators, Arch. Math. (Basel) 85 (2005), 258-267.

25. Pietsch, A., Ideals of multilinear functionals (designs of a theory), pp. 185-199 in: Proceedings of the Second International Conference on Operator Algebras, Ideals and their Applications in Theoretical Physics (Proc. Leipzig 1983), Teubner, Leipzig 1984. 
26. Zalduendo, I., Extending polynomials on Banach spaces - a survey, Rev. Un. Mat. Argentina 46 (2005), 45-72.

FACULDADE DE MATEMÁTICA

UNIVERSIDADE FEDERAL DE UBERLÂNDIA

38.400-902 - UBERLÂNDIA

BRAZIL

E-mail: botelho@ufu.br

DEPARTAMENTO DE MATEMÁTICA

INSTITUTO DE MATEMÁTICA E ESTATÍSTICA

UNIVERSIDADE DE SÃO PAULO

CAIXA POSTAL 66281

05315-970 SÃO PAULO

BRAZIL

E-mail: leonardo@ime.usp.br.
DEPARTAMENTO DE ANÁLISIS MATEMÁTICO

UNIVERSIDAD DE VALENCIA

46.100 BURJASOT - VALENCIA

SPAIN

E-mail: Pablo.Galindo@uv.es 